\title{
ENHANCEMENT IN NETWORK SECURITY WITH SECURITY PROTOCOLS
}

\author{
G.Sankareeswari ${ }^{1}$, Vidhyalakshmi $^{2}$, Selvi $^{3}$ \\ ${ }^{1,3}$ Assistant Professor, CSE, ${ }^{2}$ Assistant Professor, IT, Sri Vidya college of engineering and technology, Tamilnadu, India \\ sankariram90@gmail.com,vidhyarajme@gmail.com,sselvi201987@gmail.com
}

\begin{abstract}
Network security is a wider term used habitually to be an eternal security medium to the broadcasting environment. Cryptography, Authentication and access control Mechanisms play a vital role in secure communication over the network. The computer network is a collection of network that shares information across wired or wireless technology. In order to transfer data in a protected and confidential manner there are several security measures available. Network security can also be referred to as network safety. Network security is used to prevent the attacks by using protocols during the communication of data. This paper describes the several types of attacks, threats and protocols which attempts the secure communication between client and server.
\end{abstract}

Keywords: - DSA, DDOS, HTTPS, RADIUS, VPN, IPSEC etc...

\section{INTRODUCTION}

Network security provides the security by access control to allow only the authorized users. Network security is mainly used to prevent the attack over the network. Network security is involved in the everyday jobs conducting transactions, private sectors, government agencies and individuals. Network administrator is used to prevent and monitoring the unauthorized access, misuse and modification. network security included many cryptographic techniques which are helps to prevent the attack for improving the enhancement of the network security.

\section{WHAT IS ATTACK?}

An attack is a technique used to exploit vulnerability. There are two categories of attacks namely Passive and active attack. Passive attacks are very difficult to detect the original message but there is no possibility to modify. Example: Passive attacks are packet sniffing or traffic analysis. Active attacks are easier to detect the original message and also possibility to modify the message. Example: Active attack is denial of service.

\subsection{Plaintext and Cipher Text Attacks}

There are six related attacks over the network, including three plaintext-based methods and three cipher text-based methods:

A known plaintext attack: It is an attack where a cryptanalyst has access to a plaintext and the corresponding cipher text and find the correlation between these texts.

Cipher text-only attack: It is an attack where a cryptanalyst has access to a cipher text. It does not have access to corresponding plaintext With simple ciphers, such as the Caesar Cipher, this can be used to break the cipher by frequency analysis.

Chosen plaintext attack: It is an attack where a cryptanalyst can encrypt a plaintext of his choosing and examine the resulting cipher text. Cryptanalyst has access to a public key.

Chosen cipher text attack: It is an attack where a cryptanalyst chooses a cipher text. It deals to find a matching plaintext. This is also often performed on attacks against public key encryption; it initiates with a cipher text and find for matching plaintext data.

Adaptive Chosen Cipher text and Adaptive Chosen Plaintext Attacks: Both adaptive attacks are cryptanalyst chooses further plaintexts or cipher texts based on the results.

\section{TYPES OF ATTACKS IN AN NETWORK}

\subsection{Denial of service}

Denial of Service (DOS) attack is a type of attack over the network to disturbing the authorized use of networks, systems or applications by sending the messages which exhaust service provider's resources such as network bandwidth, system resources, and application resources.

\subsection{Spoofing (Identity Spoofing or IP Address Spoofing)}

Usually computers connected to internet by sending IP Datagram $s$ into the network. These data packets that are passed through internet carry the senders IP ADDRESS with application layer data. An attacker can gain control by using the software that runs on network device, they can easily change device protocols to replace with an arbitrary IP address Into the source address field of the data packet and this is 
called as IP Spoofing. The problem of spoofing can effectively handled by a process called Ingress Filtering is done by routers. Filtering process, the routers checks the IP address of incoming data grams and ensure that the source address that are reachable through that inter face. If it's not in the legal range, then such packets will be deleted.

\subsection{Sniffing}

The interception of data packets travelling over a network is called as packet sniffing. A sniffer program combines with network interface cards in order to confine all traffic that is travelling to and from internet host site. Through this mechanism, a sniffer that is installed in any of backbone device will now able to monitor the whole network. There are numerous sniffer programs are available on the internet at free of cost in order to make active intrusion in the network.

Sniffing can be identified by the following two ways:

Host based: This runs on individual host computers to check if the NIC is running in active mode.

Network-based: It checks for currently running process and $\log$ files.

\subsection{Mapping (Eavesdropping)}

Mapping is the process of gathering information such as IP address of computer, the operating system its running, and what are all the services available. By collecting this vital information an attacker can easily attack in focused manner. If an attacker eavesdropping (i.e. mapping the information) the network communications, then it s referred as sniffing or snooping. The process of mapping a network is a security problem which seriously considered by an enterprise.

The only solution to handle mapping is to providing strong encryption methods based on cryptography which avoid the valuable user data that are read by others when it flows through the network.

\subsection{Hijacking (Man-in-the-middle attack)}

Hijacking is a process in which an attacker between the user and the person with whom the user are communicating can be monitored, captured and controlled transparently. For example, an attacker may redirect the data exchange. This is usually happens when computers that are communicating at network layer, is unable to decide with whom they are exchanging data.

Man-in-middle attack works by recognizing the identity of the user in order to read the user message. In this manner, the person in the opposite end may believe it is you, the attacker might be actively replying as the user, and maintain the exchange continues in order to gain more information.

\subsection{Trojans}

Trojans appears to be normal software with the collection of programs, but they perform accidental or malicious actions when they are posted on the network. Spyware programs that are activated through remote control belong to this type. The Trojan file which consists of Trojan techniques appears to a standard file and standard size as a compromised system file. This type of attacks can be faced with the help of cryptographic checksum or binary digital signature procedure.

\subsection{Social Engineering}

Social engineering attack is usually carried by telephone or email message. Social engineering performs cheating to gain access to information systems. The main usage of social engineering is to involve human element in the network.

\section{MAINTAINING THE INFORMATION SECURITY:}

To enhancing the Information security, we should maintain the confidentiality, availability, integrity and authentication. Information security $=$ Confidentiality + Availability + Integrity + Authentication. The main Key to securing the information on a network is cryptography. Cryptographic terms are

Confidentiality: The ability to encrypt or encode a message to be transmitted over the network. Authentication: The ability to verify the identity of individuals or entity on the network.

Access control: The ability to control the level of access either authorized or unauthorized user. Integrity: The ability to ensure that a message or data has not been altered in transit from the sender to the recipient.

Two types of encryption techniques are available to maintain security such as,

Symmetric key Encryption: In the Symmetric key Encryption, using the private or secret key for encrypting the original message. Symmetric key cryptosystem include DES, IDEA, RC4 and SKIPJACK.

Asymmetric Key Encryption: In the Symmetric key Encryption, using the public key for encrypting the original message. Asymmetric key Encryption consist of three public key algorithms such as Diffie- Hellman, RSA and the Digital Signature Algorithm(DSA).These three Algorithms are providing enhance the security by using exchanging the key, integer factorization and digital certificates respectively.

\section{E-MAIL SECURITY:}

E-mail is always vulnerable to disclosure in one way or another. Because E-mail must traverse many networks to reach its destination During transmission, an e-mail message may pass through many mail servers. It is vulnerable to interception, replication, disclosure or modification anywhere along its prescribed path. The basic requirements of secure e- 
mail are described as follows:

Message integrity: Secure mail ensures that the message has not been modified during transmission and provides a method to certify the message's integrity by using hashing or message digest algorithm.

Verification of sender: Secure e-mail provides the cryptographic method to ensure the identity of the sender with a high degree of confidence. This is achieved by digital signature technology.

Verification of recipient: This can be achieved by employing the public key encryption techniques. The computing standards and products for secure e-mail transmission are Pretty Good Privacy (PGP), Privacy Enhanced Mail(PEM), Secure Multipurpose Internet Mail Extension(S/MIME), MIME Object Security Services(MOSS) and Message Security Protocol(MSP).use it as powerful weapon. Examples of social engineering include faked email, helpful help desk and fictitious competition.

\section{OPERATING SYSTEM SECURITY:}

Operating Systems provide the basic mechanisms for securing computer processing. It is ensuring the security that has become a mainstream issue for all operating system. If a system design does not aim for achieving the secure operating system requirements then its security features fail to protect the system.

\section{PROTOCOLS FOR NETWORK SECURITY}

\subsection{Kerberos Key Exchange}

Kerberos key exchange is a network authentication protocol. It was developed at MIT. This protocol is designed to provide the strong authentication for client/server applications by using a combination of both secret key and public key cryptography. If the Single central server utilizes the Kerberos protocol then it is referred to as a trusted server and to act as a trusted third party to authenticate users and control access to resources on the network.

\subsection{Limitations of Kerberos}

If the Kerberos server is down, one cannot access network resources, since access to all network resources must be authorized through the Kerberos server. Kerberos design is vulnerable particularly to denial of service attacks.

\subsection{Point-to-point Protocol}

PPP (Point-to-Point Protocol) is a protocol for communication between two computers using a serial interface, in general a personal computer connected by phone line to a server. It is a full-duplex protocol which can be used on different physical media and also the twisted pair or fiber optic lines or satellite transmission. It uses a variant of High Speed Data Link Control (HDLC) for packet encapsulation. a) PPP can be fragmented into three parts: Encapsulation

b) Link Control Protocol (LCP)

c) Network Control Protocol (NCP)

\subsection{Functions:}

PPP (Point-to-Point Protocol) is broadly used in the analog modem access to the ISP, where one end is PC and the other end is the ISP router. The functions are performed:

It has designed to transport multi-protocol packets between two peers connected by simple links. These links provide fullduplex concurrent bi-directional process.

\subsection{Components:}

PPP supports either asynchronous link with 8 bit of data, or with bit-oriented synchronous link. It is a method for encapsulating multi-protocol datagram's. Link Control

Protocol (LCP) is for establishing, organizing, and testing the data link connection. This allows the two ends to agree different link layer options.

The Network Control Protocols (NCP) for establishing and configuring different network-layer protocols. This permits the two ends to negotiate various network layer options.

\subsection{Operation}

To start communications over a point-to-point link and to configure and test the data link the PPP first sends LCP frames. Then the link has been recognized and facilities have been conferred as desired by the LCP. To choose and configure one or more network layer protocols the PPP sends NCP frames. From each network layer protocol packet can sent to the link. The link will remain organized for communication until LCP or NCP frames close the link.

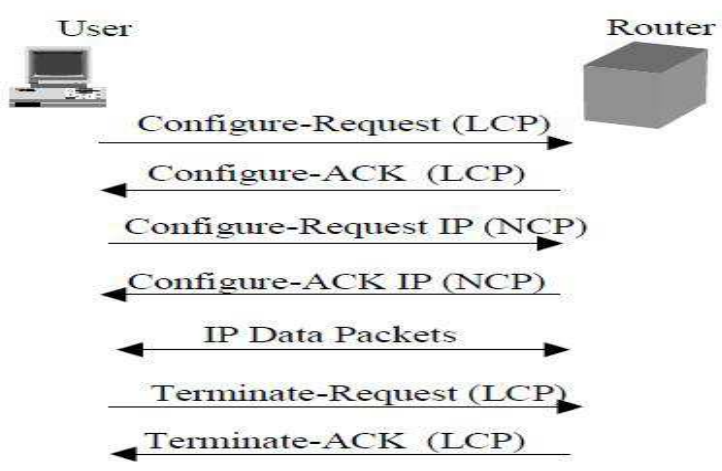

Fig 1: Sequence of the PPP Operation 
Remote authentication dial-in user service (RADIUS). RADIUS is a server for remote user authentication and accounting. It is one of the categories for Internet dial-in security protocols that include Password Authentication Protocol (PAP) and Challenge-Handshake Authentication Protocol (CHAP).To provide authentication, accounting for remote users it uses Internet Service Providers (ISPs).It also used in private networks to centralize authentication and accounting services on the network. RADIUS designed to authenticate and log dial-up remote users to a network.

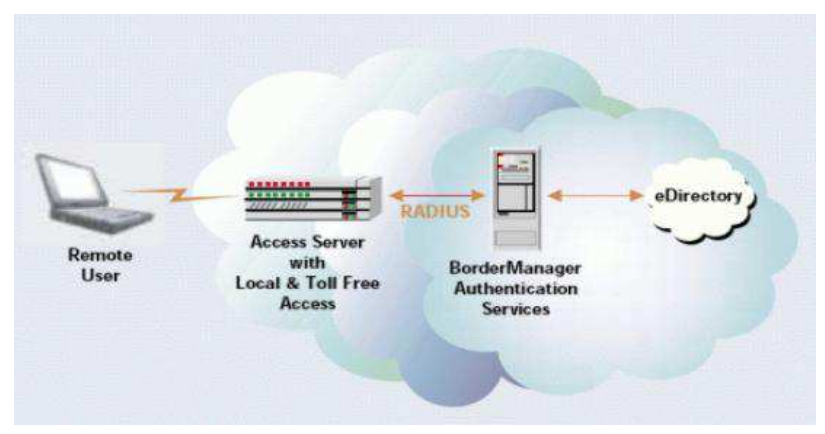

Fig 2 Remote Authentication Dial-In User Service (RADIUS)

Terminal access controller access control system( TACACS) is an authentication method that can be used to validate users who are trying to gain access to information servers, networks, and remote access servers. Three versions of the protocol: the original TACACS as just stated, XTACACS (Extended TACACS), and TACACS+.It runs as an individual third-party authentication server that gives verification services. To gain access to secure system first it prompts the user for a name and password. Then the system passes the information to the TACACS server and requests authentication services.The main protocols typically used to give Authentication, Authorization, and Accounting (AAA) services on network devices.

\subsection{TACACS+ FEATURES:}

TCP packets (port 49) guarantee that data is sent constantly across the IP network.It Supports AAA architectures and separates the each of the three AAA mechanisms. The data between the NAS and server is encrypted.

Table 1: Difference between Radius and Tacacs+

\begin{tabular}{|c|c|}
\hline RADIUS & TACACS+ \\
\hline $\begin{array}{c}\text { It combines authentication and } \\
\text { authorization. }\end{array}$ & $\begin{array}{c}\text { Separate the 3 elements of } \\
\text { AAA, } \\
\text { and it more flexible }\end{array}$ \\
\hline $\begin{array}{c}\text { Encrypts only the } \\
\text { password. }\end{array}$ & $\begin{array}{c}\text { It Encrypts both username } \\
\text { and } \\
\text { password. }\end{array}$
\end{tabular}

\begin{tabular}{|c|c|}
\hline $\begin{array}{c}\text { Requires each network device } \\
\text { to } \\
\text { contain authorization } \\
\text { configuration. } \\
\end{array}$ & $\begin{array}{l}\text { Central management for } \\
\text { authorization configuration. }\end{array}$ \\
\hline No command logging. & Complete command logging. \\
\hline $\begin{array}{c}\text { Minimal vendor support } \\
\text { for } \\
\text { authorization } \\
\end{array}$ & $\begin{array}{l}\text { Supported by most main } \\
\text { vendors. }\end{array}$ \\
\hline $\begin{array}{c}\text { Designed for subscriber } \\
\text { AAA } \\
\end{array}$ & $\begin{array}{c}\text { Designed for administrator } \\
\text { AAA }\end{array}$ \\
\hline
\end{tabular}

\section{INTERNET PROTOCOL SECURITY}

The IPSec is an encryption protocols. This protocol developed by the Internet Engineering Task Force (IETF) and designed to address of security for Internet Protocol based networks.

The latest version is IP Version 6(IPv6).This protocol perform the several services at the network layer. IPSec provides the services at the network layer: Access Control: It allows authorized user to access in order to prevent an unauthorized access to the resource. Connectionless Integrity: It is used to maintain originality of the messages and also to give an assurance that the traffic received has not been modified.

Confidentiality: It ensures that the Internet traffic is examined by the authorized parties. In Datagram data field segment that is TCP, UDP, ICMP or any other datagram data field segment are encrypted.

IPSec protocols have two protocols:

Authentication Header (AH) protocol: This protocol provides the data integrity and authentication of IP Packets.

Encapsulation Security Payload (ESP) protocol: This protocol provides data integrity, authentication and message content confidentiality

Table 2: Security at Different Level

\begin{tabular}{|c|c|}
\hline & \\
LAYER & PROTOCOLS \\
\hline APPLICATION LAYER & PGP, Kerberos, \\
& SSH etc., \\
\hline & Transport Layer \\
TRANSPORT LAYER & Security(TLS) \\
\hline & Internet Protocol \\
NETWORK LAYER & Security(IPSec) \\
\hline DATALINK LAYER & Hardware Encryption \\
\hline
\end{tabular}




\section{VIRTUAL PRIVATE NETWORKS}

VPN stands for "Virtual Private Networking. A VPN is a private network that carries the information and also proving protection by using various security mechanisms between known authorized users. In VPNs, various networking technologies are applied for providing private communications within the public telecommunication infrastructure such as the Internet. Virtual Private Network is broadly classified into four categories such as trusted VPN, Secure VPN, Hybrid VPN, Provider-provisioned VPN and two types such as, Site to site VPNs: It supports connections between two protected company networks by using ISDN, Frame Relay or ATM. Remote Access VPNs: It provides the remote access which lets single users connect to the protected company network. It provides remote access to mobile or any other resources by using internet.

\subsection{Pretty Good Privacy}

PGP stands for Pretty Good Privacy. PGP deals with encryption and decryption, it also provides an authentication for data transmission over the network. PGP is a public key cryptosystem. Secure e-mail communication is achieved by combining cryptographic algorithms. It generates the public /private pairs for secured communication. Pretty Good Privacy includes several services such as authentication, compression, confidentiality, and segmentation and E-mail compatibility.

\subsection{S/MIME}

S/ MIME stands for Secure/ Multipurpose Internet Mail Extension. This protocol is combines together the encryption and digital signature techniques. MIME deals with transfer of multimedia data (video, audio, pictures). By using DiffieHallman, RSA and Triple DES public key algorithms and session keys for transmission along with the message has been encrypted. Some of the basic services provided by S/MIME includes Privacy, data security, Authentication and Message Integrity.

\subsection{S-HTTP}

S-HTTP stands for Secure-Hyper Text Transfer Protocol. SHTTP is a secure message - oriented communications protocol. It supports certain mechanisms to provide an authentication, message integrity and confidentiality. It provides the secure communication between the client and the server in order to enable secure commercial transactions for a wide range of applications. S-HTTP messages consist of two parts. They are headers and body. S-HTTP uses headers for authentication, message encryption and digital certificates in the form of HTTP format. It contains instructions on how to decrypt the message body.

\subsection{HTTPS}

HTTPS stands for Hyper Text Transfer Protocol over Secure socket layer. It is a Web protocol. HTTPS encrypt and decrypt the user's pages in order to prevent unauthorized access. It ensures privacy and providing secure processing for several users. It provides security in online credit card processing and banking websites.

\section{CONCLUSIONS}

This paper describes the information about different attacks and protocols to prevent the attacks. Security is maintained by the different cryptographic techniques applied over the data that pass through the network. Network Security plays a vital role in the field of information systems. It protects the system connected to network by externally and internally. Furthermore, it provides effective standards and security protocols for analysis, monitoring and testing.

\section{REFERENCES}

[1] B. C. Neuman and T. Tso, "Kerberos: An Authentication Service for Computer Networks," IEEE Communications, Vol. 32, No. 9, pp. 33 - 38, Sept. 1994.

[2] S. Garfinkel, PGP: Pretty Good Privacy, O’Reilly, Dec. 1994, ISBN: 1565920988.

[3] D. E. Comer, Computer Networks and Internets, 5th Edition, Prentice Hall, Apr. 2008, ISBN: 0136061273.

[4] B. Ramsdell, S/MIME Version 3 Message Specification, IETF RFC 2633, Jun. 1999.

[5] Gray,T.,et 1(Mar.2002).Network Security credo.[Electronic version].Retrieved Nov.25,2005,from http:// staff.washington.edu/gray/papers/credo.html.

\section{BIOGRAPHIES}

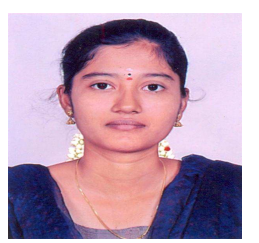

Ms. G. Sankareeswari Working as a assistant professor in Sri Vidya College of Engineering and Technology. She has completed Master of Engineering in computer science and Engineering at Mohamed Sathak Engineering College at Kilakarai. Her under graduation in Information Technology at Raja College of Engineering Technology, Madurai Her area of interest is Cloud Computing and Networks security.

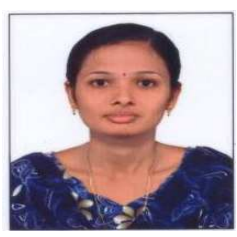

Ms. R. Vidhyalakshmi Working as a assistant professor in Sri Vidya College of Engineering and Technology. She has completed Master of Engineering in computer science and Engineering at P.S.R Engineering College at Sivakasi. Her under graduation in Information Technology at P.S.R Engineering College at Sivakasi. Her area of interest is Cloud Computing and Networks security 
Ms. S. Selvi Working as a assistant professor in Sri Vidya College of Engineering and Technology. She has completed Master of Engineering in computer science and Engineering at Coimbatore Institute of Engineering \& Technology, Coimbatore. Her under graduation in computer science and Engineering at PSR Engineering College. Sivakasi. Her area of interest is Network Security. 